\title{
Abdominal Wall Actinomycosis - A Rare Surgical Scar Infection of the Antibiotic Era
}

PETER GEORGE, ${ }^{1}$ NARASIMHA HEGDE,${ }^{1}$ JOSTOL PINTO ${ }^{2}$

\section{Case report:}

We report a 55 year old woman with abdominal wall actinomycosis after 10 years of an abdominal surgery. After a year of an elective abdominal surgery done ten years back, she noticed black discharge from the surgical scar. These lesions subsided with medications but fresh crops of discharging sinuses resulting in scars kept on erupting over the abdominal wall and extending to her back. On examination, she had a board-like abdominal wall with scars and few draining sinuses on anterior abdominal wall and lower back (figure 1 - Panel A \& B) ${ }^{1}$ Skin smears from the lesions showed branching filaments of the gram positive rod shaped bacteria and culture of the discharge showed characteristic 'molar tooth' appearance on agar, which were typical of actinomycetes (figure 1 - Panel C \& D). She responded well to penicillin and doxycycline over 24 weeks. ${ }^{2}$ Even though actinomycetes infections are rare in this era of excessive antibiotic use, a surgical scar presenting with induration or multiple discharging sinuses should arouse suspicion. ${ }^{1,2}$ Lack of familiarity with the disease and slow microbiologic confirmation techniques often result in delay of diagnosis.

\section{Conflict of Interest : None}

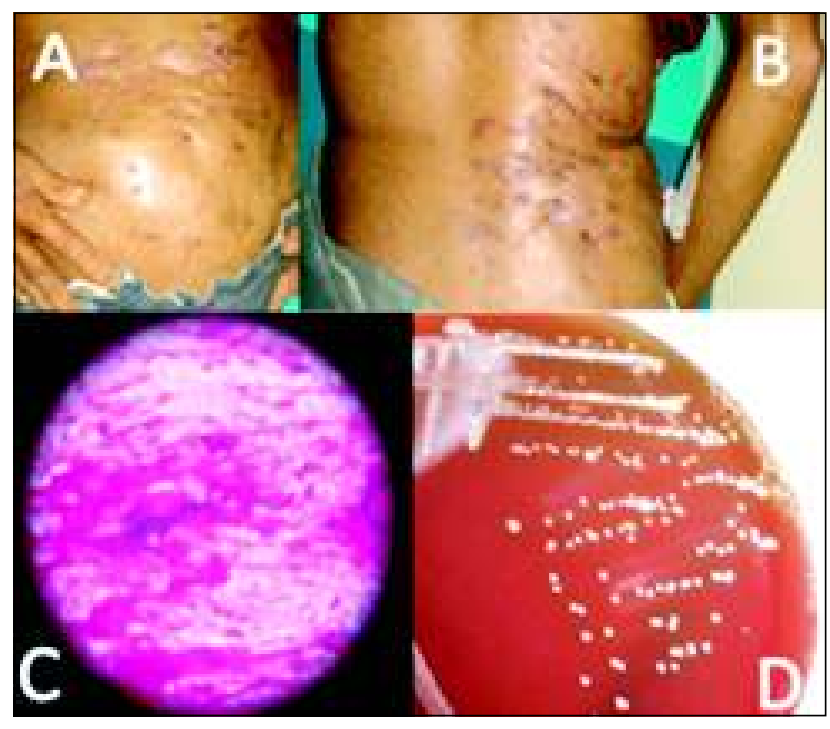

Fig.-1: Panel A \& B showing multiple cutaneous scars over the anterior abdominal wall and back. Panel C showing filaments of actinomycetes on the skin smear and panel $D$ showing typical 'molar-tooth appearance' on agar culture.

\section{References:}

1. Mueller MC, Ihrler S, Degenhart C, Bogner JR. Abdominal actinomycosis. Infection 2008; 36: 191.

2. Filipoviæ B, Miliniæ N, Nikoliæ G, Rantheloviæ T. Primary actinomycosis of the anterior abdominal wall: case report and review of the literature. J Gastroenterol Hepatol 2005; 20: 517-20.

1. Professor, Department of Medicine, Father Muller Medical College, Mangalore, India

2. Resident, Department of Medicine, Father Muller Medical College, Mangalore, India

Correspondence: Prof. Peter George, Department of Medicine, Father Muller Medical College, Mangalore, India. E-mail: drpetergeorge2002@yahoo.com 